\title{
Management of acute ulcerative and necrotising herpes simplex and zoster keratitis with amniotic membrane transplantation
}

\author{
A Heiligenhaus, H Li, E E Hernandez Galindo, J M Koch, K-P Steuhl, D Meller
}

Br J Ophthalmol 2003;87:1215-1219

\begin{abstract}
Aim: To report promoted healing of acute ulcerative and necrotising herpetic keratitis after amniotic membrane transplantation (AMT).

Methods: Retrospective, non-comparative case series of seven patients with acute ulcerative and necrotising herpetic stromal keratitis. Single or multilayer AMT with epithelial side facing up was performed. The main outcome measures were wound healing of the corneal ulcers and decrease of stromal inflammation.

Results: The mean follow up was 10.7 (SEM 1.4) months (range 5-15 months). AMT was performed once in five cases, and twice in further two. Improvement of stromal inflammation was noted within 16.4 (2.5) days (range 7-28 days). Epithelial defects healed within a mean of 17 (2.7) days (range 7-28 days). Vision improved in all but two patients. No serious side effects occurred during the follow up.

Conclusions: Although performed in an uncontrolled and non-randomised series of patients, these findings indicate that the AMT shows promise in selected cases for the restoration of ocular surface integrity, reduction of stromal inflammation, and improvement of vision in acute ulcerative and necrotising herpetic keratitis.
\end{abstract}

$\mathrm{H}$ erpetic stromal keratitis (HSK) is a common infectious cause of blindness worldwide. The development of stromal ulceration and melting is a serious complication that can lead to perforation and visual loss. The management includes anti-inflammatory medication, aggressive lubrication, tarsorrhaphy, the use of bandage contact lenses, or histoacrylate glue. Although corticosteroids are the mainstay of anti-inflammatory treatment in $\mathrm{HSK}_{1}{ }^{12}$ the intensive use of topical corticosteroids is contraindicated due to the increased risk of corneal melting or perforation. Recently, cyclosporin A has been suggested as a potential treatment option. ${ }^{3}$

Amniotic membrane (AM) is the innermost layer of the placenta and consists of a single layer of ectodermally derived amnion cells firmly attached to a basement membrane. Clinically, preserved AM used as a basement membrane substitute has been applied successfully for the management of persistent epithelial defects with and without ulcerations. ${ }^{4-9}$ Furthermore, multilayered AMT has been described to be useful for the treatment of deep corneal ulcers, descemetoceles, and small corneal perforations. ${ }^{10-13}$ Interestingly, these clinical studies indicated that corneal inflammation subsided rapidly after AMT. For instance, inflammation has been identified as a crucial factor determining the outcome of ocular surface reconstruction. ${ }^{14}$ When clinically applied as a patch, AM modulates acute inflammation. There is experimental evidence that this might be related to the expression of different anti-inflammatory proteins, ${ }^{7}$ inhibition of proteinase activity, ${ }^{15}$ exclusion of polymorphonuclear cells by subsequent apoptosis, and decrease of lipid peroxidation. ${ }^{5}$ In this context, we have also shown that AM used as a patch exhibits anti-inflammatory properties in mice with ulcerative necrotising herpetic keratitis. ${ }^{16}$ We report here our first experience in a small series of patients with acute ulcerative and necrotising herpetic stromal keratitis who were successfully treated with AMT.

\section{PATIENTS AND METHODS \\ Patients}

This study included seven eyes (seven patients) with acute ulcerative and necrotising herpetic keratitis that were managed at two different facilities from August 2000 to the end of July 2002. The patients were all referred within the first 3 weeks after the onset of keratitis. Clinical data including patient demography, aetiology, surgical procedure, subsequent surgical procedures, visual acuity, the final outcome, and complications were retrieved in a retrospective manner. All clinical data were entered into a standardised data form. The patients were evaluated after AMT by their respective surgeons. Surgical success was defined as the cessation of stromal inflammation, complete epithelialisation of the cornea, and formation of a visible stromal thickness. Failure was defined as persistence or recurrence of herpetic keratitis before the dissolution of the AM, or the lack of epithelial or stromal wound healing.

The patients' characteristics are summarised in Table 1. The diagnosis of necrotising stromal HSV keratitis was in agreement with previously published criteria. ${ }^{2}$ Briefly, patients with necrotising keratitis demonstrated an ulcer with a dense, whitish inflammatory infiltration, commonly associated with stromal oedema, and with keratic endothelial precipitates confined to the area of corneal involvement. Patients 1-3, 6, and 7 included in the study had typical dendritic or stromal herpetic keratitis of the same eye in the past. Non-necrotising keratitis presented with active inflammation and diffuse or disc-shaped oedema within the stroma, and with keratic endothelial precipitates confined to the area of corneal involvement. Patients 4 and 5 had zoster dermatitis in the ophthalmic region and keratitis in the past, as has been oberserved by the referring ophthalmologist. All patients were referred to us with corneal ulcers associated with active stromal inflammation and anterior chamber cells; a hypopyon has been seen in a single case (Table 2). Other conjunctival or corneal diseases and previous corneal surgery with the potential for corneal ulceration have been ruled out. Smears and cultures were obtained for microbiological studies at the first visitation. Significant visual deterioration was observed in all cases. At the first presentation, the 
Table 1 Amniotic membrane transplantation in seven patients with acute ulcerative and necrotising herpetic stromal keratitis. Patients' characteristics before surgery

\begin{tabular}{|c|c|c|c|c|c|c|c|}
\hline Patient No & 1 & 2 & 3 & 4 & 5 & 6 & 7 \\
\hline Sex/age (years) & $M / 76$ & $M / 43$ & $F / 63$ & $\mathrm{~F} / 60$ & $M / 63$ & $\mathrm{~F} / 72$ & $\mathrm{~F} / 59$ \\
\hline $\begin{array}{l}\text { First herpetic keratitis } \\
\text { (age, years) }\end{array}$ & 70 & 35 & 63 & 59 & 63 & 71 & 45 \\
\hline Signs of previous herpetic keratitis & $\begin{array}{l}\text { Dentritic and } \\
\text { stromal } \\
\text { ulcers }\end{array}$ & $\begin{array}{l}\text { Dendritic } \\
\text { and } \\
\text { stromal } \\
\text { ulcers }\end{array}$ & $\begin{array}{l}\text { Dendritic } \\
\text { and } \\
\text { stromal } \\
\text { ulcers }\end{array}$ & $\begin{array}{l}\text { Stromal } \\
\text { ulcer }\end{array}$ & $\begin{array}{l}\text { Stromal } \\
\text { ulcer }\end{array}$ & $\begin{array}{l}\text { Dendritic } \\
\text { ulcers }\end{array}$ & $\begin{array}{l}\text { Stromal } \\
\text { ulcer }\end{array}$ \\
\hline Previous corneal surgery & No & No & No & No & No & No & pKP \\
\hline Vision before AMT & $20 / 100$ & $\mathrm{CF}$ & $H M$ & $H M$ & LP & CF & HM \\
\hline
\end{tabular}

$A M T=$ amniotic membrane transplantation; $C F=$ counting fingers; $H M=$ hand motion, $L P=$ light perception, pKP= perforating keratoplasty

patients were treated with topical (four times daily) and systemic $(5 \times 400 \mathrm{mg}$ daily $)$ aciclovir and lubricants. They did not receive a bandage contact lens or tarsorrhaphy. The amount of stromal thinning varied between 30\% and $90 \%$. Size and localisation of the corneal ulcers are shown in Table 2. A descemetocele was noted in the patient No 4. Even though a dense stromal inflammatory cell infiltration with deep ulceration was present in all patients, no perforation was seen (Table 2). Since corneal ulcers and inflammation were refractory to this medical treatment within the first week, AMT was considered.

\section{Human amniotic membrane preparation}

Human AM has been prepared as previously described. ${ }^{4}$ Briefly, human AM was processed after elective caesarean delivery when blood borne micro-organisms such as human immunodeficiency virus types 1 and 2, hepatitis virus type B and $\mathrm{C}$, and syphilis had been excluded by serological tests. Hepatitis virus type $C$ was additionally excluded by means of polymerase chain reaction. The authors (AH and JK; DM and KPS) procured and preserved AM, and screened the donors at their respective facilities, following the same protocol.

\section{The AMT procedure}

All the procedures followed the tenets of the Declaration of Helsinki. Informed consent was obtained before the surgery. Patients were anaesthetised either with peribulbar or retrobulbar block. Depending on the depth of the corneal ulcer, a single or multilayer technique ${ }^{10-12}$ was chosen. Briefly, the base of the corneal ulcer was cleaned off the necrotic tissue, and the poorly adherent epithelium adjacent to the ulcer was removed.

Several pieces of AM were trimmed to fit the shape and the size of the corneal ulcer and were then placed in the defect with basement membrane side facing up. AM were secured with interrupted 10-0 Nylon sutures. Finally, a large piece of AM was used as a temporary patch in patients 1, 2, and 6 extending over the entire limbus, and was finally anchored on the cornea with a running 10-0 Nylon suture to the perilimbal episclera. In these patients, a bandage contact lens was finally applied on the AM.

Postoperative medication included topical gentamicin, hyaluronic acid, and $0.1 \%$ dexamethasone, each three times daily. Systemic $(5 \times 400 \mathrm{mg}$ daily) aciclovir was continued for 1 month, and $2 \times 400 \mathrm{mg}$ were given for further 5 months. Patients were followed up frequently with respect to the visual acuity, slit lamp appearance, and applanation tonometry. All complications from AMT were documented.

\section{RESULTS}

The follow up period ranged from 7 to 15 months (mean 10.7 (SEM 1.4) months) after AMT. The AM used as a patch dissolved quickly under the bandage contact lens during a period of 1-6 weeks (mean 3.2 weeks). A second AMT was necessary in two patients (Nos 1 and 5), because the corneal ulceration was still present after the first membrane has resolved. Persistent ulcerative keratitis was observed, but successfully managed by repeating AMT. In patient 5, the single layered AM resolved very fast. Repeated AMT was combined with a tarsorrhaphy and punctal occlusion. In another patient (No 2), the ulcerative herpetic keratitis recurred 2 weeks after the multilayered AMT was resolved. Therefore, a second multilayered AMT was performed in this patient. In patient No 4 with a deep corneal ulcer and descemetocele, corneal perforation did not develop after multilayered AMT was performed. The results after AMT are summarised in Table 3.

Eventually, complete epithelial closure was obtained in all of the patients (see Fig 1). The time between AMT and epithelial healing varied between 7 and 28 days (mean 17 (SEM 2.7) days). The healing of stromal inflammation was noted in all of the seven treated eyes between the days 7 and 28 after AMT (mean $16.4(2.5)$ ). The improvement of stromal

Table 2 Amniotic membrane transplantation in seven patients with acute ulcerative and necrotising stromal herpetic keratitis. Slit lamp appearance before surgery

\begin{tabular}{|c|c|c|c|c|c|c|c|}
\hline Patient No & 1 & 2 & 3 & 4 & 5 & 6 & 7 \\
\hline Size of ulcer $(\mathrm{mm})$ & 3 & 3 & 4 & 5 & 4.5 & $2 \times 8$ & 3 \\
\hline Localisation of ulcer & Periphery & $\begin{array}{l}\text { Central } \\
\text { Mid-periphery }\end{array}$ & Central & Central & Central & Periphery & Mid-periphery \\
\hline Stromal thinning (\%) & 30 & 30 & 30 & 90 & 20 & 50 & 30 \\
\hline Descemetocele & No & No & No & Small & No & No & No \\
\hline Perforation & No & No & No & No & No & No & No \\
\hline $\begin{array}{l}\text { Dense stromal cell } \\
\text { infiltration }\end{array}$ & Yes & Yes & Yes & Yes & Yes & Yes & Yes \\
\hline AC cells & Yes & Yes & Yes & Yes & Yes & Yes & Yes* \\
\hline
\end{tabular}

$\mathrm{AMT}=$ amniotic membrane transplantation; $\mathrm{AC}=$ anterior chamber; *hypopyon. 
Table 3 Amniotic membrane transplantation (AMT) in seven patients with acute ulcerative and necrotising herpetic stromal keratitis. Surgical procedures and results after surgery

\begin{tabular}{|c|c|c|c|c|c|c|c|}
\hline Patient No & 1 & 2 & 3 & 4 & 5 & 6 & 7 \\
\hline Number of AMTs & 2 & 1 & 1 & 1 & 2 & 1 & 1 \\
\hline AMT technique & Multilayer & Multilayer & Single layer & Multilayer & Single layer & Multilayer & Single layer \\
\hline Follow up (months) & 11 & 14 & 8 & 15 & 8 & 7 & 14 \\
\hline $\begin{array}{l}\text { Epithelial healing after } \\
\text { AMT (days) }\end{array}$ & 21 & 7 & 21 & 17 & 14 & 28 & 11 \\
\hline $\begin{array}{l}\text { Stromal healing after } \\
\text { AMT (days) }\end{array}$ & 14 & 7 & 21 & 17 & 14 & 14 & 28 \\
\hline Vision after AMT & $20 / 30$ & $20 / 100$ & $20 / 200$ & $20 / 200$ & $H M$ & $20 / 100$ & $\mathrm{HM}$ \\
\hline Vision improved after AMT & Yes & Yes & Yes & Yes & Yes & Yes & No \\
\hline Complications after AMT & No & No & No & No & No & No & No \\
\hline Other surgical procedures & No & No & No & Botulinum & $\mathrm{T}, \mathrm{PO}$ & No & No \\
\hline
\end{tabular}

keratitis correlated with a decrease of the anterior chamber inflammation (Table 3, Fig 1).

In this series of patients, no postoperative complication occurred from AMT, as superinfection, new appearance of descemetocele, perforation, or hypopyon were not seen. Recurrent herpetic keratitis after dissolution of the AM was seen in three of the seven patients. This was noted in the weeks 2 and 12, and between 2 and 5 months after the AM dissolved, respectively. The intraocular pressures were within normal ranges throughout the study period.

In five of the seven patients the vision increased two or more lines after the AMT in comparison with the preoperative values. Owing to the severity of keratitis and the central localisation of the ulcers, the final visual acuity was $20 / 100$ or worse in six of the seven cases. In all patients, the ulcerative corneas healed with dense scar formation and irregular corneal thickness, which eventually also affected the visual outcome depending on their localisation. Furthermore, cataracts that reduced the final vision significantly were present in patients 1 , and 3-7.

\section{Case report}

Patient 6

A 72 year old white woman had sudden redness and visual loss in her right eye. The medical history was remarkable for herpetic dendritic ulcers. A recurrence of stromal herpetic keratitis was diagnosed. When a l week treatment with topical aciclovir, lubricants, and antibiotics failed, she was referred to the infirmary. The vision was CF. There was an initially $30 \%$ deep peripheral corneal ulceration $(2 \times 8 \mathrm{~mm})$ with marked conjunctival inflammation. A dense, whitish inflammatory infiltration associated with marked stromal oedema was present, with keratic endothelial precipitates confined to the area of corneal involvement that extended to the central cornea (Fig 1). This was accompanied by severe iritis. The intraocular pressure was with $15 \mathrm{~mm} \mathrm{Hg}$ in the normal range. Bacterial and parasitic infections were ruled out by smears and cultures. Systemic aciclovir was started at a dosage of $5 \times 400 \mathrm{mg}$ daily, ofloxacin eye drops were given five times daily, and lubricants were given hourly. Since the corneal ulcer progressed to 50\% depth within l week, amniotic membrane transplantation was performed. Under retrobulbar anaesthesia, the necrotic tissue was removed from the corneal ulcer, and the poorly adherent epithelium adjacent to the ulcer was removed. Several pieces of AM were placed in the deep ulcer, and were fixed with 10-0 Nylon sutures. This was covered with a large piece of AM, which was anchored to the adjacent conjunctiva and cornea. A bandage contact lens was applied on the AM (Fig 1). Topical gentamicin, hyaluronic acid, and $0.1 \%$ dexamethasone were given after the surgery. Aciclovir was continued at $5 \times 400 \mathrm{mg}$ daily for 1 month, and $2 \times 400 \mathrm{mg}$ were given for five more
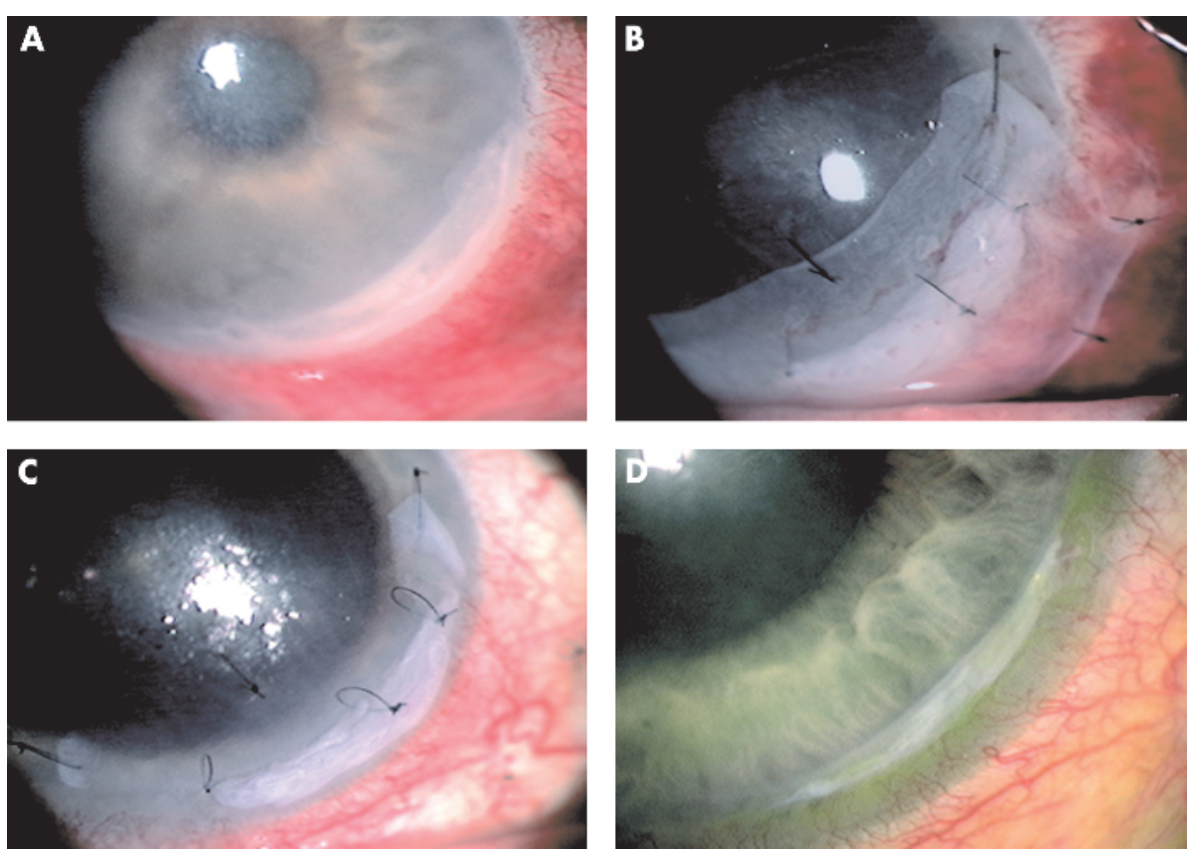

Figure 1 Patient with herpetic keratitis complicated by deep peripheral ulcerative keratitis and anterior uveitis. (A) Preoperative slit lamp appearance. (B) Two days after multilayer amniotic membrane transplantation. (C) Two weeks after surgery; the superficial layer of $A M$ is nearly resorbed; deep layers of $A M$ in the stroma; the epithelial defect is nearly closed; marked reduction of stromal inflammation and uveitis. (D) Two months after surgery: fluorescein staining shows that the epithelium is closed. The stromal defect is filled with AM. No stromal inflammation or uveitis, no neovascularisation. 
months. There was an immediate relief of the patient's discomfort after surgery. At day 14 after surgery, stromal inflammation and iritis disappeared, and the AM has been nearly dissolved (Fig 1). The deep layers of AM in the stroma were visible, and the epithelial defect was nearly closed. Complete epithelialisation was noted on day 28. Two months after surgery AM was detected under the epithelium (Fig 1D). At the last follow up visit 7 months after surgery, vision was 20/200 as a result corneal haze and cataract formation. Disease recurrence or complications have not been seen after surgery.

\section{DISCUSSION}

The anti-inflammatory action of AM has been observed in vitro and in animal models, and corneal ulcers have already been successfully treated with amniotic membrane transplantation. ${ }^{10-12}{ }^{15-17}$ In this series with seven patients, the herpes simplex virus or varicella zoster induced severe ulcerative keratitis healed rapidly after AMT.

The viral ulcers healed in five of the seven eyes after the first AMT, and in a further two patients after a second AMT. The stromal defect was filled up with AM, if necessary with multiple layers of AM. Additionally, the entire defect was covered with AM in an effort to provide as much AM tissue as possible. Although the nature of the anti-inflammatory action of AMT is still undefined, it has been speculated that the improvement of herpetic ulcers is attributed to soluble factors released from the AM. The critical action mechanisms related to the AM might involve promotion of epithelialisation, inactivation of proteolytic enzymes, inactivation of neutrophils, secretion of growth factors, or the downregulation of pro-inflammatory cytokines. ${ }^{5} 781015161819$

AMT for the treatment of corneal epithelial defects and stromal ulcers has not been suggested as first or second line therapy by most of the physicians, but has been reserved for cases in which the medical options, a bandage contact lens or tarsorrhaphy, have failed. ${ }^{20}$ Most recently, Kim and coworkers $^{13}$ have reported on a large series of patients with infectious keratitis, including seven cases with herpetic keratitis, that were successfully treated with AMT when the medical treatment failed. Our preliminary observations support their notion that AMT may be an initial surgical option in selected cases with deep ulcers from herpes viruses. However, only some of the patients in the study from Kim et al seemed to have active inflammatory keratitis at the time of surgery, as reflected by the good final visual outcome. In contrast, all of the patients in this study had severe and active necrotising inflammation. The grade of inflammatory activity of our patients is reflected by the notion that anterior chamber cells were present in all of them.

The action mechanism of AMT to improve ulcerative keratitis has not been studied here. However, AMT may not work primarily as a contact lens that improves corneal wetting, since frequent use of tear substitutes has not been successful before. In patient No 5, the single layered AM resolved very fast, probably because of a highly inflamed ocular surface and poor eyelid blinking. Both factors together dramatically reduced the ocular defence and, therefore, repeated AMT was combined with a tarsorrhaphy and punctal occlusion.

An extensive microbiological examination must be performed at the initial step, and we suggest that appropriate antiviral and antimicrobial drugs should be instituted at least several days before AMT. In cases with progressing ulcerative necrotising keratitis AMT might then be considered. The other well established anti-inflammatory and antiviral therapies should then be continued for the long term management.
Serious complications were not noted in our series. However, a close follow up is mandatory after performing AMT on an inflamed cornea with ulceration. Although visualisation of the cornea is impaired by the whitish AM, the corneal stroma and anterior chamber could be sufficiently judged with the slit lamp in our patients.

Recurrence of the ulcerative herpetic process occurred in three patients in this series. This is in accordance with the findings in a recent experimental study that herpetic stromal keratitis rapidly improved after AMT but recurred after AMT removal. ${ }^{16}$ Consequently, it appears that the anti-inflammatory effects of AMT are transient and decrease after AMT dissolution. These observations underline the fact that antiviral and anti-inflammatory drugs are indispensable in these patients.

The final vision noted in this series was poor and correlated with the severity of disease. However, perforation of the globe was avoided and inflammation was arrested by AMT. It is our impression that corneal neovascularisation, which is generally noticeable after use of histoacrylate glue for management of corneal ulcers, was less frequently observed after AMT. Previous experimental data have proposed that AM exhibits anti-angiogenic properties. ${ }^{7}$ This may be beneficial to decreasing the risk of rejection in the event of subsequent corneal transplantation.

Although it has been speculated that AMT for the management of acute infectious keratitis has a high risk of superinfection or HSV reactivations, this was not seen in this series. However, the adjunctive use of antibiotics and antivirals with AMT must be recommended. It is likely that AMT soaked with antimicrobial drugs is functioning as a depot for drug delivery, in a similar way to a collagen shield.

The design of our study does not allow a judgment about the value of AMT in comparison with other treatment regimens. The preliminary findings here need further confirmation in a controlled and randomised study.

\section{ACKNOWLEDGEMENTS}

We appreciate the help in the study by Stefan Kremmer, MD, University of Essen.

\section{Authors' affiliations \\ A Heiligenhaus, $\mathbf{H}$ Li, J M Koch, Department of Ophthalmology, at St Franziskus Hospital, Muenster, Germany \\ E E Hernandez Galindo, K-P Steuhl, D Meller, Department of Ophthalmology, University of Essen, Essen, Germany \\ Supported in part by DAAD-FUNDAYACUCHO (A/99/25975) Bonn, Germany.}

Correspondence to: Arnd Heiligenhaus, MD, Department of Ophthalmology, St Franziskus Hospital, Hohenzollernring 74, 48145 Muenster, Germany; arnd.heiligenhaus@t-online.de

Accepted for publication 6 February 2002

\section{REFERENCES}

1 Power WJ, Hillery MP, Benedict-Smith A, et al. Acyclovir ointment plus betamethasone or placebo in first episode disciform keratitis. $\mathrm{Br} J$ Ophthalmol 1992;76:711-13.

2 Wilhelmus KR, Gee L, Hauck WW. Herpetic eye disease study. A controlled trial of topical corticosteroids for herpes simplex stromal keratitis. Ophthalmology 1994;101:1883-96.

3 Heiligenhaus A, Steuhl KP. Topical cyclosporin A in patients with herpes simplex stromal keratitis. Graefes Arch Clin Exp Ophthalmol 1999;237:435-8.

4 Lee S, Tseng SCG. Amniotic membrane transplantation for persistent epithelial defects with ulceration. Am J Ophthalmol 1997;123:303-12.

5 Park WC, Tseng SCG. Modulation of acute inflammation and keratocyte death by suturing, blood and amniotic membrane in PRK. Invest Ophthalmol Vis Sci 2000:41:2906-14. 
6 Shimazaki J, Yang HY, Tsubota K. Amniotic membrane transplantation for ocular surface reconstruction in patients with chemical and thermal burns. Ophthalmology 1997; 104:2068-76.

7 Hao Y, Hui-Kang D, Hwang DG, et al. Identification of antiangiogenic and antiinflammatory proteins in human amniotic membrane. Cornea 2000;19:348-52

8 Shimmura S, Shimazaki J, Ohashi Y, et al. Antiinflammatory effects of amniotic membrane transplantation in ocular surface disorders. Cornea 2001;20:408-13.

9 Meller D, Pires RT, Mack RJ, et al. Amniotic membrane transplantation for acute chemical or thermal burns. Ophthalmology 2000;107:980-9.

10 Hanada K, Shimazaki J, Shimmura S, et al. Multilayer amniotic membrane transplantation for severe ulceration of the cornea and sclera. Am J Ophthalmol 2001;131:324-31.

11 Kruse FE, Rohrschneider K, Voelcker HE. Multilayer amniotic membrane transplantation for reconstruction of deep corneal ulcers. Ophthalmology 1999; 106:1504-11

12 Solomon A, Meller D, Prabhasawat $\mathrm{P}$, et al. Amniotic membrane grafts for non-traumatic corneal perforations, descemetoceles and deep ulcers. Ophthalmology 2002; 109:694-703.
$13 \mathrm{Kim}$ JS Kim JC, Hahn TW et al Amniotic membrane transplantation in infectious corneal ulcers. Cornea $2001 ; 20: 720-6$

14 Tseng SC, Tsubota K. Important concepts for treating ocular surface and tear disorders. Am J Ophthalmol 1997; 124:825-35.

$15 \mathrm{Kim} \mathrm{JS}, \mathrm{Kim} \mathrm{JC}, \mathrm{Na} \mathrm{BK}$, et al. Amniotic membrane patching promotes healing and inhibits proteinase activity on wound healing following acute corneal akali burn. Exp Eye Res 2000;70:329-37.

16 Heiligenhaus A, Baver D, Meller D, et al. Improvement of HSV-1 necrotizing keratitis with amniotic membrane transplantation. Invest Ophthalmol Vis Sci 2001:42:1969-74.

17 Chen HJ, Pires RT, Tseng SC. Amniotic membrane transplantation for severe neurotrophic corneal ulcers. Br J Ophthalmol 2000;84:826-33.

18 Solomon A, Rosenblatt M, Monroy D, et al. Suppression of interleukin- $1 \alpha$ and interleukin-1 $\beta$ in human limbal epithelial cells cultured on the amniotic membrane stromal matrix. Br J Ophthalmol 2001;85:444-9.

19 Bültmann S, You L, Spandau U, et al. Amniotic membrane down-regulates chemokine expression in human keratocytes. Invest Ophthalmol Vis Sci 1999:40:S578.

20 Letko E, Stechschulte SU, Kenyon KR, et al. Amniotic membrane inlay and overlay grafting for corneal epithelial defects and stromal ulcers. Arch Ophthalmol 2001;119:659-63.

\section{The lighter side}

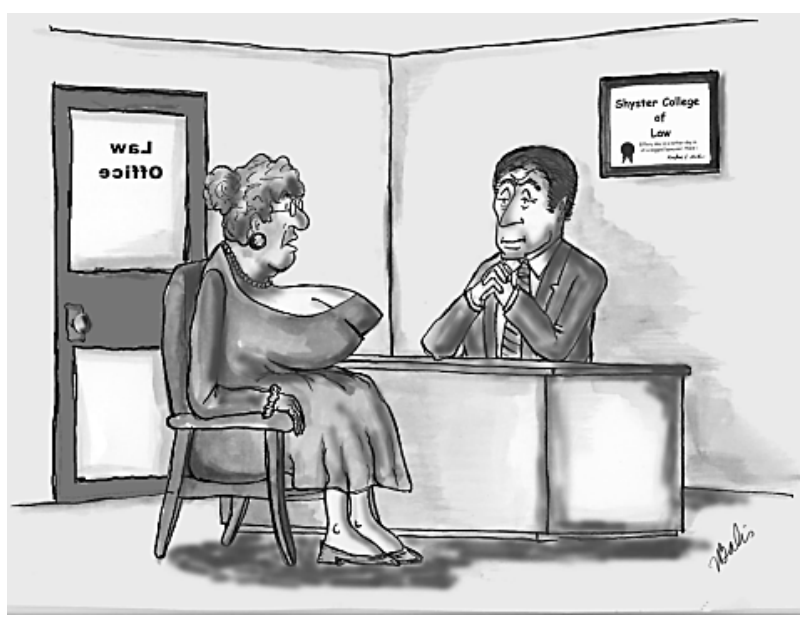

If I understand you correctly, you expected lens implants and you were wheeled into the wrong room. (c) Michael Balis. 